\title{
Beihilfe zum Suizid - ein ergreifender Bericht zu komplexen Streitfragen
}

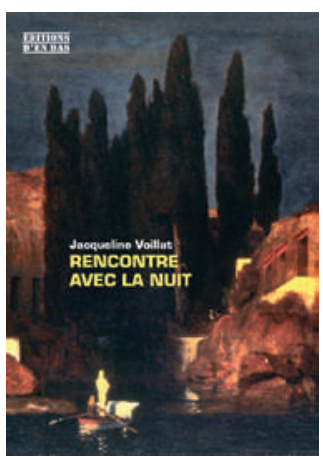

Umschlag des Buches von Jacqueline Voillat [2].
1 Nationale Ethikkommission. Beihilfe zum Suizid. Stellungnahme Nr. 9/2005, Empfehlung 4.

www.nek-cne.ch

2 Voillat J. Rencontre avec la nuit. Lausanne: Editions d'en bas; 2012, 148 S.

3 Rezension des Buches: S. Arsever. Le Temps (Genf), 12. Juni 2012.
Im Mai 2011 wurde im Kanton Zürich zweimal über Suizidhilfe und mögliche Änderungen in Bezug auf den liberalen, offenen Art. 115 StGB abgestimmt (für einen Suizidenten geleistete Hilfe ist nicht strafbar, es sei denn, sie geschähe aus selbstsüchtigen Beweggründen). Die waadtländischen Bürger stimmten am 17. Juni dieses Jahres für Art. $27 \mathrm{~d}$ des Gesundheitsgesetzes, der mit folgenden Worten eingeleitet wird (Originaltext französisch): «Die Einrichtungen des Gesundheitswesens von öffentlichem Interesse können eine vom Patienten verlangte Beihilfe zum Suizid unter ihrem Dach nicht ablehnen, wenn folgende Bedingungen erfüllt sind ...» (der Entscheid selbst muss von einem urteilsfähigen Patienten kommen, der ernsthaft und unheilbar erkrankt ist).

Unsere Bevölkerung will ganz offenkundig für jeden die Freiheit wahren, über sich selbst verfügen zu können. Zwar hat der Bundesrat zweimal entschieden, dass der Status quo (Art. 115 StGB ohne weitere rechtliche Ergänzung) sehr wohl das weniger schlechte System sein könnte; doch die Debatte geht weiter und betrifft die Gesellschaft und die Ethik auf sozialer, beruflicher und individueller Ebene. Ich halte es für sehr wichtig, dass der Staat nie den Eindruck vermittelt, er heisse den Suizid gut, Suizid sei einfach eine Option unter vielen. Staatlicherseits verfügte, ergänzende Anordnungen laufen Gefahr, so interpretiert werden zu können, als legitimierten sie den Suizid (dies könnte auch für die neue waadtländische Rechtsnorm gelten). Die Erfahrung hat mich gelehrt, dass es Dinge gibt, die weder der Staat noch das Gesetz adäquat regeln kann, vor allem wenn es um zutiefst persönliche Fragen geht. In dieser Sache kann es keine «optimale», für alle gültige Haltung geben. Jeder einzelne Fall - so auch die nationale Ethikkommission [1] - ist getrennt zu betrachten, in seinen unterschiedlichen Dimensionen, mit den betroffenen Personen, im Dialog mit den Angehörigen (inkl. Arzt und Pflegepersonal).

Die Einzelschicksale sind alle verschieden ... Ein jüngst veröffentlichtes Buch verdient dabei Erwähnung [2]: Es handelt sich um die Beschreibung einer Familie (hochbetagte Mutter und zwei Töchter) und deren Auseinandersetzung mit der immer wieder gelegentlich aggressiv - vorgebrachten Forderung der Mutter, Exit in Anspruch nehmen zu wollen. Mit Flashbacks auf vorausgehende Jahrzehnte fokussiert das Buch vor allem auf die sechs Monate, die die alte Dame in der Geriatrie verbringt, nachdem sie ihre Wohnung verlassen musste. Die Autorin, eine der Töchter, beschreibt in allen Einzelheiten die Bezie- hung zwischen der Mutter, ihrer Schwester und sich selbst. Es kommt zu vielen heftigen Wortwechseln auf der Grundlage eines Familienlebens, das zwar seine Schwierigkeiten hatte, dem die Schwestern jedoch durch schöne Erinnerungen verbunden bleiben. Beschrieben werden auch die Kontakte und Diskussionen mit den Ärzten und dem Pflegepersonal, aus denen hervorgeht, dass wir zwar nicht in der perfekten Welt leben und einige Fachkräfte weniger gute Beziehungen pflegen als andere, im allgemeinen letztlich jedoch alle bemüht sind, ihre Rollen korrekt und nach den Regeln des Standes zu erfüllen. Dennoch gibt es zahlreiche Augenblicke der Spannung und auch der Wut. Dazwischen liegen Zögerlichkeiten und viele Fragen, sowohl philosophischer als auch praktischer Natur.

Letztlich wird Exit gerufen. Ein Arzt der Vereinigung trifft sich mehrmals mit der Patientin und ihren Töchtern, ebenso mit dem Spitalarzt. Man einigt sich auf die Sterbehilfe, und der Termin wird drei Wochen im Voraus festgesetzt. Nach den Turbulenzen und Ambivalenzen rund um die Forderung der alten Dame ist die Darstellung der Gefühle der Protagonisten während dieser letzten Phase bewegend vor allem unmittelbar vor und nach dem Ableben der Mutter. Wichtige Hilfe leistet auch der Pastor des Wohnorts. Er besucht die Beteiligten und ist beim Suizid anwesend. Im Nachwort gesteht J. Voillat, dass sie drei Jahre gebraucht habe, um diese Geschichte niederzuschreiben. Sie dankt den Mitarbeitenden im Gesundheitsdienst, die sich mit einer ungewöhnlichen Situation und dem beharrlichen Willen der Patientin konfrontiert sahen. «Ich bewundere, dass sie ihre Prinzipien und Zweifel überwinden und den so starken Wunsch meiner Mutter akzeptieren konnten, dass sie sie bis zum Ende umsorgt haben, respektvoll und vielleicht mit Zuneigung.»

Die Autorin ist Lehrerin und hat ganz gewiss Talent zum Schreiben. Dieses Buch beleuchtet die Problematik der Suizidhilfe und die damit verknüpften Herausforderungen, denen sich die Betroffene und ihre Nächsten zu stellen hatten. Um es mit den Worten einer klugen Leserin auszudrücken: Ein Buch «über die Zweifel, die Zerreissproben und letztlich das sichere Wissen in einem atemberaubenden Kampf (...) ein Bericht, der kein Plädoyer sein will und sich allen Lesarten öffnet» [3].

Jean Martin, ehemaliger Kantonsarzt, Redaktionsmitglied und Mitglied der Nationalen Ethikkommission 